\title{
Chattering-Free Sliding Mode Control for Networked Control System with Time Delay and Packet Dropout Based on a New Compensation Strategy
}

\author{
Yu Zhang ${ }^{\mathbb{D}},{ }^{1}$ Shousheng Xie, ${ }^{1}$ Ledi Zhang, ${ }^{1}$ Litong Ren, ${ }^{2}$ Bin Zhou ${ }^{D},{ }^{1}$ and Hao Wang ${ }^{1}$ \\ ${ }^{1}$ Aeronautics and Astronautics Engineering Institute, Air Force Engineering University of PLA, Xian 710038, China \\ ${ }^{2}$ Unit 94314 of Chinese PLA, Zhengzhou 450003, China \\ Correspondence should be addressed to Yu Zhang; frank_sharon1314@126.com
}

Received 25 March 2019; Revised 19 June 2019; Accepted 1 July 2019; Published 11 July 2019

Academic Editor: Yong Xu

Copyright (c) 2019 Yu Zhang et al. This is an open access article distributed under the Creative Commons Attribution License, which permits unrestricted use, distribution, and reproduction in any medium, provided the original work is properly cited.

\begin{abstract}
This paper addresses the sliding mode control problem for a class of networked control systems with long time delay and consecutive packet dropout. A new modeling method is proposed, through which time delay and packet dropout are modeled in a unified model described by one Markov chain. To avoid the chattering problem of classic reaching law, a new chattering-free reaching law is proposed. Then with a focus on the problem that controller-actuator channel network condition cannot be foreseen by the controller, a new compensation strategy is proposed, which can effectively compensate for the effect of time delay and packet dropout in controller-actuator channel. Finally, a simulation example is presented to demonstrate the effectiveness of the proposed approach.
\end{abstract}

\section{Introduction}

In recent years, a lot of interest and concern have been focused on networked control systems (NCSs) where communication channels interconnect sensors with controllers and/or controllers with actuators [1]. Compared with conventional point-to-point interconnected feedback control systems, NCSs have advantages in many aspects: higher system operability, more efficient resource utilization and sharing, lower cost, and reduced weight, as well as simplicity for system installation and maintenance [2-4]. Despite these distinctive features provided by NCSs, the insertion of communication network in the feedback control loops also brings about new challenges such as bandwidth-limited channel, network-induced delay, packet dropout, and packet disordering [5].

Time delay and packet dropout serve as two of the most common and crucial problems of NCSs that can directly degrade the performance of the closed-loop system or even lead to instability [6]. More specifically, long time delay refers to the time delay larger than the sampling period which, compared with short time delay or "one step" time delay, can cause more serious problems like packet disordering. Therefore, for discrete-time systems, it is more valuable to carry out research on long time delay systems. Numerous effective methods have been reported in the literature aiming to tackle the time delay and packet dropout problems arising in NCSs (see, for example, [7-10]). It can be concluded that the solution of the problem consists of two main parts, one of which is to establish an appropriate model to describe time delay and packet dropout and the other is to design a controller that can guarantee system stability despite the existence of time delay and packet dropout. This paper is also organized based on this structure.

Firstly, since time delay and packet dropout usually exist simultaneously in the real network, it is important to establish a model to handle them in a common framework. Various modeling methods have been proposed including time delay system model [11], switched system model [12-14], asynchronous dynamical system model $[15,16]$, and stochastic system model $[6,8,9,17-25]$. Among them, stochastic system model becomes popular in recent years and one of the most 
used ways is to adopt a Markov discrete-time linear system to describe random time delay or packet dropouts $[9,20-$ $22,24,25]$. It is found that the transition from one time delay/packet dropout state to another usually occurs with a certain probability and thus a Markov chain can be used to describe such relation with a transition probability matrix. Therefore, long time delay and consecutive packet dropout can be modeled as a Markov chain. In [24], both sensor-tocontroller and controller-to-actuator delays are considered and described by Markov chains and the resulting closedloop systems are written as jump linear systems with two modes. Similarly, in [25], the characteristics of network communication delays and packet dropouts in both sensor-tocontroller and controller-to-actuator channels are modeled and their history behavior is described by three Markov chains. However, it is discovered that most of the existing Markov chain based modeling methods have a common problem that the time delay and packet dropout are usually considered on the controller side, which means they do not reflect the condition of the packets actually received by the actuator. This could lead to the problem of the use of outdated signals and packet disordering. To avoid this problem, buffer or zero-order-holder ( $\mathrm{ZOH})$ are introduced [26], but pitifully, the model used has not changed in [26]. Similarly, ZOH is also used in [27] and a corresponding modeling method is proposed, but [27] considers only the problem of packet dropout. Motivated by the above discussions, this paper focuses on proposing a new model that considers both long time delay and consecutive packet dropout in a unified model, and the situation of actual received control signals on the actuator side will be considered to avoid problems like packet disordering and the use of obsolete data.

When modeling method is determined, the next key problem is to design an appropriate controller that can guarantee system stability and performance of the NCSs despite the existence of time delay and packet dropout. Among various methods, sliding mode control (SMC) method stands out for its unique feature that system states can be driven onto a specially designed sliding surface and then become totally insensitive to uncertainties [28-30]. However, the well-known chattering problem limits it wide application especially in discrete systems. With a focus on this problem, this paper proposes a chattering-free sliding mode reaching law for a type of multiple-input NCSs, and it needs to be pointed out that, to the best of the author's knowledge, the use of such chattering-free reaching law in multiple-input systems has rarely been reported.

Moreover, another critical problem usually ignored is that the network condition of the controller-actuator channel cannot be foreseen by the controller when calculating the control signal, which makes the calculated control signal not suitable for the systems when there exists time delay or packet dropout. Some researchers have noticed this problem but they tried to solve the problem only by using the time delay and packet dropout information of the former sampling period as compensation [31]. Therefore, another novelty of this paper is that it proposes a new compensation strategy, where all possible time delay and packet dropout states in the controller-actuator channel are considered and a multiplemodel based controller is designed which can output a sequence of control signals, and then a compensator on the actuator side will decide which signal of the control signal sequence to use according to the time stamp information. In this way, the time delay and packet dropout can be properly compensated for even when the network condition of controller-actuation channel cannot be foreseen.

The main contributions of this paper can be concluded as follows: (i) a new modeling method is proposed for NCSs with long time delay and consecutive packet dropout; (ii) a chattering-free sliding mode reaching law is proposed and first used for multiple-input NCSs; (iii) a multiple-model based sliding mode controller is designed together with a new compensation strategy which can compensate for time delay and packet dropout without the advanced knowledge of controller-actuator channel network condition.

The remainder of this paper is organized as follows. Section 2 introduces the newly proposed NCSs modeling method. The design of chattering-free sliding mode controller as well as the new compensation strategy is presented in Section 3. Numerical simulation results are shown in Section 4 . Section 5 presents some conclusions.

\section{A New Modeling Method for NCSs with Time Delay and Packet Dropout}

2.1. Modeling of Time Delay and Packet Dropout. Consider the discrete NCSs with time delay and packet dropout, which is described by the following state space model:

$$
\boldsymbol{x}(k+1)=\boldsymbol{A} \boldsymbol{x}(k)+\boldsymbol{B} \boldsymbol{u}(k)+\boldsymbol{d}(k),
$$

where $\boldsymbol{x}(k) \in \boldsymbol{R}^{n}$ is system state variable; $\boldsymbol{u}(k) \in \boldsymbol{R}^{m}$ is control input; $\boldsymbol{d}(k) \in \boldsymbol{R}^{n}$ is external disturbance; and $\boldsymbol{A}, \boldsymbol{B}$, and $\boldsymbol{C}$ are matrices with appropriate dimensions.

To make it easier for analysis, the following assumptions are made for system (1).

Assumption 1. System (1) is controllable and all system state variables are observable.

Assumption 2. Time delay and packet dropout exist in the controller-actuator channel only and each data packet is sent with a time stamp.

Assumption 3. The long time delay $\tau$ is bounded and the upper bound is known, which is denoted as $\bar{\tau}$; consecutive packet dropout is also bounded and the largest number of consecutive packet dropouts is known, which is denoted as $\bar{\rho}$.

Assumption 4. External disturbance $\boldsymbol{d}(k)$ is unknown but bounded and satisfies sliding mode matching condition, i.e.,

$$
\boldsymbol{d}(k)=\boldsymbol{B} \boldsymbol{\omega}(k) .
$$

Now we are going to consider long time delay and consecutive packet dropout for system (1). We will first establish a time delay model based on Markov chain, then we will take packet 


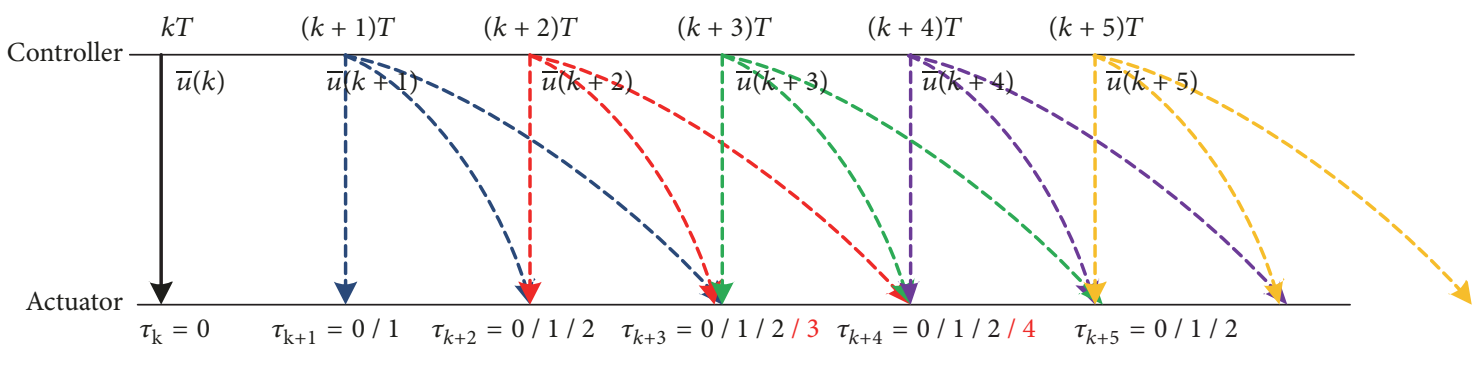

FIGURE 1: Signal transmission timing diagram with $\tau_{\max }=2, \rho_{\max }=2$.

dropout as a special type of time delay, and the obtained transition probability matrix will be extended. Such modeling method considers the connection between time delay and packet dropout on the actuator side, which makes the model established better reflect the real condition in practical use.

Inspired by [27], a ZOH is adopted for the NCS. Therefore, due to the existence of $\mathrm{ZOH}$, only the newest received control signal will be used. Here we assume that time delay $\tau(k)$ takes values in the set $\boldsymbol{\Omega}_{\tau}=\{0,1,2, \ldots \bar{\tau}\}$. It is noticed from the value space of $\tau(k)$ that time delay can be zero in our model because, for a normal NCS, it is not possible that there is no on-time arriving signal. Therefore, it is more reasonable to add zero to the value space of time delay. First, let us think about an example, in which $\tau(k)=0$. It means controller output signal $\overline{\boldsymbol{u}}(k)$ arrives at time $k$, so the actuator input $\boldsymbol{u}(k)=\overline{\boldsymbol{u}}(k)$. Then, due to the existence of $\mathrm{ZOH}$, there are only two possible conditions for $\boldsymbol{u}(k+1)$. If $\overline{\boldsymbol{u}}(k+1)$ also arrives on time, we have $\boldsymbol{u}(k+1)=\overline{\boldsymbol{u}}(k+1)$ and $\tau(k+1)=0$. If $\overline{\boldsymbol{u}}(k+1)$ does not arrive on time, then $\overline{\boldsymbol{u}}(k)$ will still be used as an actuator input and we have $\boldsymbol{u}(k+1)=\overline{\boldsymbol{u}}(k)$ and $\tau(k+1)=1$. In the same way, if we assume the time delay of time instant $k$ is $\tau(k)=i(\mathrm{i}<\bar{\tau})$, there could be only $i+2$ possible conditions for the next time instant; i.e., if the control signal of $k+1$ arrives on time, then $\tau(k+1)=0$; if the control signal of $k+1$ does not arrive but the signal sent out at time $k$ arrives, then we have $\tau(k+1)=1$; if control signal of $k+1$ or $k$ does not arrive but that of time $k-1$ arrives, then we have $\tau(k+1)=2$; the same way can explain the conditions all the way to $\tau(k+1)=i$; however, if there is no newly arriving signal at $k+1$, then, due to the existence of $\mathrm{ZOH}$, the control signal of time $k$ is still used, so the value of time delay becomes $\tau(k+1)=i+1$. In particular, when $\tau(k)=\bar{\tau}$, which is the upper bound of time delay, there could only be $\bar{\tau}+1$ conditions. The existence of $\mathrm{ZOH}$ guarantees the use of newest signal, which means, for example, if control signal $\overline{\boldsymbol{u}}(k+1)$ arrives at time instance $k+1$, then all signals sent before time instant $k+1$ will never be used afterwards. The time delay state transition can be described as

$$
\begin{aligned}
\pi_{i j} & =\operatorname{Pr}(\tau(k+1)=j \mid \tau(k)=i) \\
\sum_{j=0}^{\bar{\tau}} \pi_{i j} & =1
\end{aligned}
$$

where $i \in \boldsymbol{\Omega}_{\tau}, j \in\{0,1, \ldots, i+1\} \cap \boldsymbol{\Omega}_{\tau}$, and define $\Pi_{\bar{\tau}}=\pi_{i j}$ as system time delay transition possibility matrix, which is given as follows:

$$
\begin{aligned}
& \Pi_{\bar{\tau}} \\
& =\left[\begin{array}{cccccc}
\pi_{00} & \pi_{01} & 0 & 0 & \cdots & 0 \\
\pi_{10} & \pi_{11} & \pi_{12} & 0 & \cdots & 0 \\
\vdots & \vdots & \vdots & \ddots & \ddots & \vdots \\
\pi_{(\bar{\tau}-2) 0} & \pi_{(\bar{\tau}-2) 1} & \pi_{(\bar{\tau}-2) 2} & \cdots & \pi_{(\bar{\tau}-2)(\bar{\tau}-1)} & 0 \\
\pi_{(\bar{\tau}-1) 0} & \pi_{(\bar{\tau}-1) 1} & \pi_{(\bar{\tau}-1) 2} & \cdots & \pi_{(\bar{\tau}-1)(\bar{\tau}-1)} & \pi_{(\bar{\tau}-1) \bar{\tau}} \\
\pi_{\bar{\tau} 0} & \pi_{\bar{\tau} 1} & \pi_{\bar{\tau} 2} & \cdots & \pi_{\bar{\tau}(\bar{\tau}-1)} & \pi_{\bar{\tau} \bar{\tau}}
\end{array}\right] .
\end{aligned}
$$

Then let us take consecutive packet dropout into consideration. As mentioned above, if there is only bounded time delay, then when $\tau(k)=\bar{\tau}$, there could be only $\bar{\tau}+1$ possible conditions. However, if packet dropout is also considered, the time delay condition of time $k+1$ is different but can also be included and described in a similar way.

Firstly, an example with $\tau_{\max }=2$ and $\rho_{\max }=2$ is shown in Figure 1 as follows to illustrate the situation when consecutive packet dropout is included.

It is shown that $\overline{\boldsymbol{u}}(k)$ is transmitted to the actuator at time instant $k$ without time delay, thus giving $\tau(k)=0$. Then at next time instant $k+1$, as illustrated above, $\tau(k+1)$ could only be $\tau(k+1)=0$ or 1 . If $\tau(k+1)=1$, then at time instant $k+2$, we have $\tau(k+2)=0$, or 1 , or 2 . If $\tau(k+2)=2$, since time delay is bounded, then, at time instant $k+3$, the late arriving signal could only be $\overline{\boldsymbol{u}}(k+1)$ or $\overline{\boldsymbol{u}}(k+2)$, and if there is still no newly arrived signal, it is certain that packet $\overline{\boldsymbol{u}}(k+1)$ and the signals sent before $k+1$, if still not received, are already lost. On this occasion, $\mathrm{ZOH}$ outputs control signal $\overline{\boldsymbol{u}}(k)$ as actuator input and the equivalent time delay is $\tau(k+3)=3$. If $\tau(k+3)=3$, since it is sure that $\overline{\boldsymbol{u}}(k+1)$ is already lost, then, at time instant $k+4$, the late arriving signal could only be $\overline{\boldsymbol{u}}(k+2)$ or $\overline{\boldsymbol{u}}(k+3)$ and if there is still no newly arrived signal, $\overline{\boldsymbol{u}}(k)$ will still be used by the actuator; thus the equivalent time delay is $\tau(k+4)=4$. If $\tau(k+4)=4$, since consecutive packet dropout is also bounded, $\tau(k+5)$ could only take values in the set $\{0,1,2\}$. Extend the above discussion to $\tau_{\max }=\bar{\tau}, \rho_{\max }=\bar{\rho}$ and define the value space of equivalent time delay as $\boldsymbol{\Omega}_{\tau}=$ 
$\{0,1,2, \ldots,(\bar{\tau}+\bar{\rho})\}$; then the following equivalent time delay transition is given:

$$
\begin{aligned}
\pi_{i j} & =\operatorname{Pr}(\tau(k+1)=j \mid \tau(k)=i) \\
\sum_{j=0}^{\bar{\tau}+\bar{\rho}} \pi_{i j} & =1
\end{aligned}
$$

where $i \in \boldsymbol{\Omega}_{\tau}, j \in\{0,1, \ldots, i+1\} \cap \boldsymbol{\Omega}_{\tau} \cup\{i+1\}$, and define $\Pi_{\bar{\tau}, \bar{\rho}}=\pi_{i j}$ as system equivalent time delay transition possibility matrix, which is given as follows:

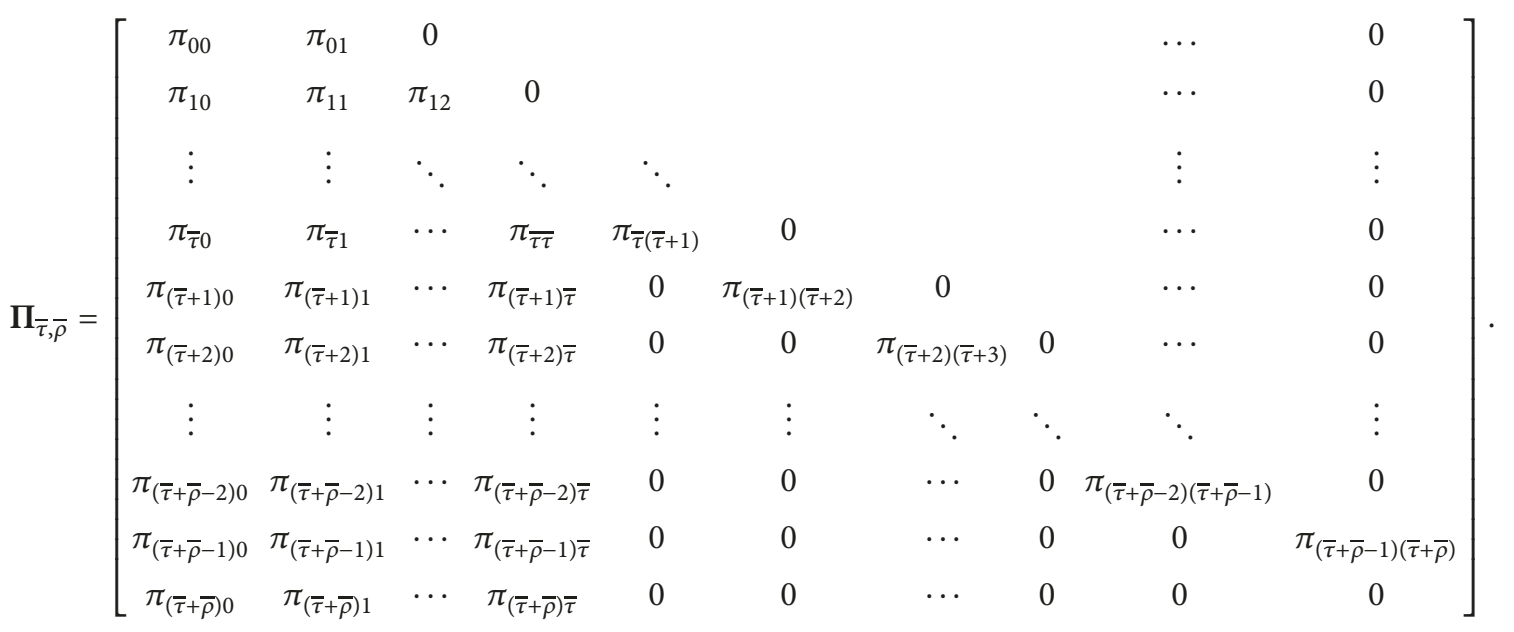

As a result, the NCSs with long time delay and consecutive packet dropout can be expressed as

$$
\boldsymbol{x}(k+1)=\boldsymbol{A} \boldsymbol{x}(k)+\boldsymbol{B} \boldsymbol{u}(k-\tau(k))+\boldsymbol{d}(k),
$$

where $\tau(k)$ is equivalent time delay.

Remark 5. The proposed new modeling method stands out from other traditional stochastic system modeling methods in the following three aspects:

(i) It innovatively models long time delay and consecutive packet dropout in a unified model described by only one Markov chain.

(ii) The time scale adopted in our Markov chain is linear with the physical time; i.e., the state transition in our Markov chain always happens over one physical time instant.

(iii) Compared with traditional models, the transition probability matrix of our model is not a full matrix and thus it requires less work in obtaining the transition probability matrix.

2.2. Elimination of Time Delay Term in the Form. First, according to Assumption 4, system (7) can be rewritten as

$$
\boldsymbol{x}(k+1)=\boldsymbol{A} \boldsymbol{x}(k)+\boldsymbol{B}[\boldsymbol{u}(k-\tau(k))+\boldsymbol{\omega}(k)] .
$$

The following linear transformation can be adopted to obtain a new expression of system (8) which does not have time delay term in the form. When $\tau(k) \geq 1$, by defining

$$
\overline{\boldsymbol{x}}(k)=\boldsymbol{A}^{\tau} \boldsymbol{x}(k)+\sum_{i=0}^{\tau-1} \boldsymbol{A}^{\tau-i-1} \boldsymbol{B}[\boldsymbol{u}(k-\tau+i)+\boldsymbol{\omega}(k)],
$$

system (8) can be transformed as

$$
\overline{\boldsymbol{x}}(k+1)=\boldsymbol{A} \overline{\boldsymbol{x}}(k)+\boldsymbol{B}[\boldsymbol{u}(k)+\boldsymbol{\omega}(k)] .
$$

According to Assumption 1, system (10) is also controllable.

Proof. The term $\boldsymbol{B}[\boldsymbol{u}(k-\tau(k))+\boldsymbol{\omega}(k)]$ can be decomposed as follows:

$$
\begin{aligned}
\boldsymbol{B}[\boldsymbol{u}(k-\tau)+\boldsymbol{\omega}(k)] \\
=\boldsymbol{B}[\boldsymbol{u}(k-\tau)+\boldsymbol{\omega}(k)] \\
\quad-\boldsymbol{A}^{-1} \boldsymbol{B} \boldsymbol{u}[(k-\tau+1)+\boldsymbol{\omega}(k)] \\
\quad+\boldsymbol{A}^{-1} \boldsymbol{B}[\boldsymbol{u}(k-\tau+1)+\boldsymbol{\omega}(k)]-\cdots \\
\quad-\boldsymbol{A}^{-\tau+1} \boldsymbol{B}[\boldsymbol{u}(k-1)+\boldsymbol{\omega}(k)] \\
+\boldsymbol{A}^{-\tau+1} \boldsymbol{B}[\boldsymbol{u}(k-1)+\boldsymbol{\omega}(k)] \\
\quad-\boldsymbol{A}^{-\tau} \boldsymbol{B}[\boldsymbol{u}(k)+\boldsymbol{\omega}(k)]+\boldsymbol{A}^{-\tau} \boldsymbol{B}[\boldsymbol{u}(k)+\boldsymbol{\omega}(k)] \\
=\boldsymbol{A}^{-\tau} \boldsymbol{B}[\boldsymbol{u}(k)+\boldsymbol{\omega}(k)] \\
\quad+\sum_{i=0}^{\tau-1} \boldsymbol{A}^{-i} \boldsymbol{B}[\boldsymbol{u}(k-\tau+i)+\boldsymbol{\omega}(k)] \\
\quad-\sum_{i=0}^{\tau-1} \boldsymbol{A}^{-i-1} \boldsymbol{B}[\boldsymbol{u}(k-\tau+1+i)+\boldsymbol{\omega}(k)] .
\end{aligned}
$$


Substituting (11) into (8), we have

$$
\begin{gathered}
\boldsymbol{x}(k+1)+\sum_{i=0}^{\tau-1} \boldsymbol{A}^{-i-1} \boldsymbol{B}[\boldsymbol{u}(k-\tau+1+i)+\boldsymbol{\omega}(k)] \\
=\boldsymbol{A}\left[\boldsymbol{x}(k)+\sum_{i=0}^{\tau-1} \boldsymbol{A}^{-i-1} \boldsymbol{B}[\boldsymbol{u}(k-\tau+i)+\boldsymbol{\omega}(k)]\right] \\
+\boldsymbol{A}^{-\tau} \boldsymbol{B}[\boldsymbol{u}(k)+\boldsymbol{\omega}(k)] .
\end{gathered}
$$

Premultiplying both sides of (12) by $\boldsymbol{A}^{\tau}$, we have

$$
\begin{aligned}
\boldsymbol{A}^{\tau} \boldsymbol{x}(k+1)+\sum_{i=0}^{\tau-1} \boldsymbol{A}^{\tau-i-1} \boldsymbol{B}[\boldsymbol{u}(k-\tau+1+i)+\boldsymbol{\omega}(k)] \\
=\boldsymbol{A}\left[\boldsymbol{A}^{\tau} \boldsymbol{x}(k)+\sum_{i=0}^{\tau-1} \boldsymbol{A}^{\tau-i-1} \boldsymbol{B}[\boldsymbol{u}(k-\tau+i)+\boldsymbol{\omega}(k)]\right] \\
+\boldsymbol{B}[\boldsymbol{u}(k)+\boldsymbol{\omega}(k)] .
\end{aligned}
$$

Finally let $\overline{\boldsymbol{x}}(k)=\boldsymbol{A}^{\tau} \boldsymbol{x}(k)+\sum_{i=0}^{\tau-1} \boldsymbol{A}^{\tau-i-1} \boldsymbol{B}[\boldsymbol{u}(k-\tau+i)+\boldsymbol{\omega}(k)]$; system (10) can obtained. In particular, when $\tau(k)=0$, it is defined that $\overline{\boldsymbol{x}}(k)=\boldsymbol{x}(k)$.

\section{Design of Chattering-Free Sliding Mode Controller}

3.1. Design of Linear Sliding Surface. Since disturbance satisfies the matching condition, then when designing the sliding surface, the system can be transformed to a sliding mode regular form so that the sliding mode dynamic is totally free from the disturbance. To obtain the sliding mode regular form of (10), we can define a nonsingular matrix $\boldsymbol{T} \in \mathrm{R}^{n \times n}$, which makes

$$
\boldsymbol{T} \boldsymbol{B}=\left[\begin{array}{c}
0_{(n-m) \times m} \\
\boldsymbol{B}_{m}
\end{array}\right],
$$

where $\boldsymbol{B}_{m} \in \boldsymbol{R}^{m \times m}$ is nonsingular. Choose $\boldsymbol{T}=\left[\begin{array}{ll}\boldsymbol{T}_{2} & \boldsymbol{T}_{1}\end{array}\right]^{T}$, where $\boldsymbol{T}_{1} \in \boldsymbol{R}^{n \times m}, \boldsymbol{T}_{2} \in \boldsymbol{R}^{n \times(n-m)}$ are two subblocks of a unitary matrix resulting from the singular value decomposition of $\boldsymbol{B}$, i.e.,

$$
\boldsymbol{B}=\left[\begin{array}{ll}
\boldsymbol{T}_{1} & \boldsymbol{T}_{2}
\end{array}\right]\left[\begin{array}{c}
\boldsymbol{\Sigma}_{m \times m} \\
0_{(n-m) \times m}
\end{array}\right] \boldsymbol{J}^{\mathrm{T}},
$$

where $\boldsymbol{\Sigma}$ is a diagonal positive-definite matrix and $\boldsymbol{J}$ is a unitary matrix.

Then through linear transformation $\boldsymbol{z}=\boldsymbol{T} \overline{\boldsymbol{x}}$, system (10) can be decomposed into two subsystems:

$$
\begin{aligned}
\boldsymbol{z}_{1}(k+1)= & \widehat{\boldsymbol{A}}_{11} \boldsymbol{z}_{1}(k)+\widehat{\boldsymbol{A}}_{12} \boldsymbol{z}_{2}(k), \\
\boldsymbol{z}_{2}(k+1)= & \widehat{\boldsymbol{A}}_{21} \boldsymbol{z}_{1}(k)+\widehat{\boldsymbol{A}}_{22} \boldsymbol{z}_{2}(k) \\
& +\boldsymbol{B}_{m}[\boldsymbol{u}(k)+\boldsymbol{\omega}(k)],
\end{aligned}
$$

where $\boldsymbol{z}_{1}(k) \in \boldsymbol{R}^{n-m}, \boldsymbol{z}_{2}(k) \in \boldsymbol{R}^{m} ; \widehat{\boldsymbol{A}}=\boldsymbol{T} \boldsymbol{A} \boldsymbol{T}^{-1}$.
It has been proved that (16a) is the sliding mode dynamics of system (10).

Choose the following classic linear sliding surface:

$$
\boldsymbol{s}(k)=\boldsymbol{C} \boldsymbol{z}(k)=\left[\begin{array}{ll}
\boldsymbol{C}_{1} & \boldsymbol{I}
\end{array}\right] \boldsymbol{z}(k)=\boldsymbol{C}_{1} \boldsymbol{z}_{1}(k)+\boldsymbol{z}_{2}(k) .
$$

In the sliding mode, we have

$$
\begin{aligned}
\boldsymbol{s}(k) & =\boldsymbol{C}_{1} \boldsymbol{z}_{1}(k)+\boldsymbol{z}_{2}(k)=0 \Longrightarrow \\
\boldsymbol{z}_{2}(k) & =-\boldsymbol{C}_{1} \boldsymbol{z}_{1}(k) .
\end{aligned}
$$

Substituting (18) into (16a), we have

$$
z_{1}(k+1)=\left(\widehat{A}_{11}-\widehat{A}_{12} C_{1}\right) z_{1}(k),
$$

which is a classic sliding surface design problem, and the sliding surface parameter $\boldsymbol{C}$ can be obtained through pole placement.

3.2. Design of Chattering-Free SMC. To suppress chattering, a new chattering-free sliding mode reaching law is proposed, inspired by [32]. However, the reaching law proposed in this paper is for multiple-input systems, which means sliding surface $\boldsymbol{s}(k)$ is not a scalar but a vector, and the reaching law should be newly defined. The vector form reaching law is given bellow:

$$
\boldsymbol{s}(k+1)=\boldsymbol{s}(k)-q T \boldsymbol{s}(k)-\varepsilon T \operatorname{sig}^{\alpha}(\boldsymbol{s}(k)),
$$

where $0<q T<1,0<\varepsilon T<1,0<\alpha<1$ and,

$$
\begin{aligned}
\operatorname{sig}^{\alpha}(\boldsymbol{s}(k)) \triangleq & \operatorname{diag}\left\{\left|\boldsymbol{s}_{1}(k)\right|^{\alpha},\left|\boldsymbol{s}_{2}(k)\right|^{\alpha}, \ldots,\left|\boldsymbol{s}_{m}(k)\right|^{\alpha}\right\} \\
& \cdot \operatorname{sgn}(\boldsymbol{s}(k)) .
\end{aligned}
$$

Therefore, the sliding mode controller can be designed by comparing (16a), (16b), and (20):

$$
\begin{aligned}
\boldsymbol{u} & (k)=\left(-\boldsymbol{B}_{m}\right)^{-1}\left[\boldsymbol{C}_{1} \widehat{\boldsymbol{A}}_{11} \boldsymbol{z}_{1}(k)+\boldsymbol{C}_{1} \widehat{\boldsymbol{A}}_{12} \boldsymbol{z}_{2}(k)\right. \\
& +\widehat{\boldsymbol{A}}_{21} \boldsymbol{z}_{1}(k)+\widehat{\boldsymbol{A}}_{22} \boldsymbol{z}_{2}(k)-(1-q T) \boldsymbol{s}(k) \\
& \left.+\varepsilon T \operatorname{sig}^{\alpha}(\boldsymbol{s}(k))+\boldsymbol{B}_{m} \boldsymbol{\omega}(k)\right] .
\end{aligned}
$$

It can be seen that the unknown disturbance term is included in the expression of $\boldsymbol{u}(k)$, so $\boldsymbol{u}(k)$ cannot be used directly as a control signal. To tackle this problem we introduce the following disturbance estimation strategy:

$$
\begin{aligned}
\widehat{\boldsymbol{\omega}}(k)= & \boldsymbol{z}_{2}(k)-\widehat{\boldsymbol{A}}_{21} \boldsymbol{z}_{1}(k-1)-\widehat{\boldsymbol{A}}_{22} \boldsymbol{z}_{2}(k-1) \\
& -\boldsymbol{B}_{m} \boldsymbol{u}(k-1)=\boldsymbol{\omega}(k-1) .
\end{aligned}
$$

Substituting (23) into (22) yields the final SMC law:

$$
\begin{aligned}
\boldsymbol{u}(k) & =\left(-\boldsymbol{B}_{m}\right)^{-1}\left[\boldsymbol{C}_{1} \widehat{\boldsymbol{A}}_{11} \boldsymbol{z}_{1}(k)+\boldsymbol{C}_{1} \widehat{\boldsymbol{A}}_{12} \boldsymbol{z}_{2}(k)\right. \\
+ & \widehat{\boldsymbol{A}}_{21} \boldsymbol{z}_{1}(k)+\widehat{\boldsymbol{A}}_{22} \boldsymbol{z}_{2}(k)-(1-q T) \boldsymbol{s}(k) \\
& \left.+\varepsilon T \operatorname{sig}^{\alpha}(\boldsymbol{s}(k))+\boldsymbol{B}_{m} \widehat{\boldsymbol{\omega}}(k)\right] .
\end{aligned}
$$


With the SMC law (24), the new sliding mode reaching law considering disturbance is

$$
\begin{aligned}
\boldsymbol{s}(k+1)= & (1-q T) \boldsymbol{s}(k)-\varepsilon T \operatorname{sig}^{\alpha}(\boldsymbol{s}(k)) \\
& +\boldsymbol{B}_{m}(\boldsymbol{\omega}(k)-\widehat{\boldsymbol{\omega}}(k)) \\
= & (1-q T) \boldsymbol{s}(k)-\varepsilon T \operatorname{sig}^{\alpha}(\boldsymbol{s}(k)) \\
& +\boldsymbol{B}_{m}(\boldsymbol{\omega}(k)-\boldsymbol{\omega}(k-1)) .
\end{aligned}
$$

Since $\boldsymbol{s}(k+1)$ is a vector with $m$ sliding mode functions, then we write (25) into the following componentwise form and we will prove that each sliding mode state satisfies the reaching condition. The componentwise form of (25) is

$$
\begin{aligned}
s_{i}(k+1)= & (1-q T) s_{i}(k)-\varepsilon T\left|s_{i}(k)\right|^{\alpha} \operatorname{sgn}\left(s_{i}(k)\right) \\
& +\boldsymbol{w}_{i}\left(\omega_{i}(k)-\omega_{i}(k-1)\right) \\
& (i=1,2, \ldots, m),
\end{aligned}
$$

where $\boldsymbol{w}_{i}$ is the $i$ th row of $\boldsymbol{B}_{m}$, i.e.,

$$
\boldsymbol{B}_{m}=\left[\begin{array}{c}
\boldsymbol{w}_{1} \\
\vdots \\
\boldsymbol{w}_{m}
\end{array}\right] .
$$

Assumption 6. The change rate of disturbance $\delta_{i}(k)=\omega_{i}(k)-$ $\omega_{i}(k-1)$ is limited, and there exists a constant $\delta^{*}$ such that $\max \left|w_{i} \delta_{i}(k)\right| \leq \delta^{*}, \quad(i=1,2, \ldots, m)$.

Theorem 7. With the constant matrix $\mathbf{C}$ and the linear sliding surface given by (17), the trajectory of system sliding mode state $s_{i}(k)(i=1,2, \ldots, m)$ can be driven by controller (25) into the sliding surface neighborhood region $\mathbf{\Omega}$ within at most $m_{i}^{*}$ steps, where

$$
\begin{gathered}
\mathbf{\Omega}=\left\{s_{i}(k)|| s_{i}(k) \mid \leq \eta=\xi(\alpha)\right. \\
\left.\cdot \max \left\{\left(\frac{\delta^{*}}{\varepsilon T}\right)^{1 / \alpha},\left(\frac{\varepsilon T}{1-q T}\right)^{1 /(1-\alpha)}\right\}\right\} \\
\xi(\alpha)=1+\alpha^{\alpha /(1-\alpha)}-\alpha^{1 /(1-\alpha)}
\end{gathered}
$$

$$
m_{i}^{*}=\left[m_{i}\right]+1
$$

$$
\text { with } m_{i}=\frac{s_{i}^{2}(0)-\eta^{2}}{\mu^{2}} \mu=q T \eta+1-q T \text {. }
$$

Proof. The proof of the theory consists of two problems:

(i) we need to prove that system sliding mode state will enter the region $\boldsymbol{\Omega}$ within $m_{i}^{*}$ steps.

(ii) we will prove that if $s_{i}(k) \in \Omega$, then $s_{i}(k+1) \in \Omega$.

Proof of Problem (i). Define a Lyapunov function $V_{i}(k)=$ $s_{i}^{2}(k)$; then it is obtained that

$$
\begin{aligned}
& \Delta V_{i}(k)=s_{i}^{2}(k+1)-s_{i}^{2}(k)=-\left[q T s_{i}(k)\right. \\
& \left.\quad+\varepsilon T\left|s_{i}(k)\right|^{\alpha} \operatorname{sgn}\left(s_{i}(k)\right)-w_{i} \delta_{i}(k)\right] \cdot\left[2 s_{i}(k)\right. \\
& \left.\quad-q T s_{i}(k)-\varepsilon T\left|s_{i}(k)\right|^{\alpha} \operatorname{sgn}\left(s_{i}(k)\right)+w_{i} \delta_{i}(k)\right] .
\end{aligned}
$$

Then the following two conditions are discussed.

(1) If $s_{i}(k)>\eta$, there is

$$
\begin{aligned}
\Delta V_{i}(k)= & -\left[q T s_{i}(k)+\varepsilon T s_{i}^{\alpha}(k)-w_{i} \delta_{i}(k)\right] \\
& \cdot\left[2 s_{i}(k)-q T s_{i}(k)-\varepsilon T s_{i}^{\alpha}(k)+w_{i} \delta_{i}(k)\right] .
\end{aligned}
$$

Since $s_{i}(k)>\eta$, there is no doubt that $s_{i}(k)>\xi(\alpha) \cdot\left(\delta^{*} / \varepsilon T\right)^{1 / \alpha}$. Thus, we have

$$
\begin{aligned}
& q T s_{i}(k)+\varepsilon T s_{i}^{\alpha}(k)-w_{i} \delta_{i}(k) \\
& \quad \geq q T s_{i}(k)+\xi^{\alpha}(\alpha) \delta^{*}-\left|w_{i} \delta_{i}(k)\right| \\
& \quad \geq q T \cdot \eta+\left[\xi^{\alpha}(\alpha)-1\right] \delta^{*} .
\end{aligned}
$$

Define $\mu=q T \cdot \eta+\left[\xi^{\alpha}(\alpha)-1\right] \delta^{*}$, and then since $0<q T<1$, $1<\xi^{\alpha}(\alpha)<2$, it is sure that $\mu$ is a positive constant.

Similarly, since $s_{i}(k)>\eta$, there is no doubt that $s_{i}(k)>$ $\eta=\xi(\alpha) \cdot(\varepsilon T /(1-q T))^{1 /(1-\alpha)}$. Thus, we have

$$
(1-q T) s_{i}(k)>\xi(\alpha)^{1-\alpha} \cdot \varepsilon T .
$$

Therefore,

$$
(1-q T) s_{i}(k)>\xi^{1-\alpha}(\alpha) \cdot \varepsilon T s_{i}^{\alpha}(k)>\varepsilon T s_{i}^{\alpha}(k) .
$$

By further calculation, we have

$$
s_{i}(k)>q T s_{i}(k)+\varepsilon T s_{i}^{\alpha}(k) .
$$

Then it can be obtained that

$$
\begin{aligned}
& 2 s_{i}(k)-q T s_{i}(k)-\varepsilon T s_{i}^{\alpha}(k)+w_{i} \delta_{i}(k) \\
& \geq q T s_{i}(k)+\varepsilon T s_{i}^{\alpha}(k)+w_{i} \delta_{i}(k) \\
& \geq q T s_{i}(k)+\varepsilon T s_{i}^{\alpha}(k)-\left|w_{i} \delta_{i}(k)\right| \geq \mu .
\end{aligned}
$$

Consequently, there is

$$
\begin{aligned}
\Delta V_{i}(k)= & -\left[q T s_{i}(k)+\varepsilon T-w_{i} \delta_{i}(k)\right] \\
& \cdot\left[2 s_{i}(k)-q T s_{i}(k)-\varepsilon T+w_{i} \delta_{i}(k)\right] \leq-\mu^{2} \\
< & 0 .
\end{aligned}
$$

(2) If $s_{i}(k)<-\eta$, by a similar proof procedure, it can be shown that the relation $\Delta V_{i}(k) \leq-\mu^{2}$ still holds. Therefore, when $s_{i}(k) \notin \Omega, \Delta V_{i}(k)=s_{i}^{2}(k+1)-s_{i}^{2}(k) \leq-\mu^{2}<0$.

Moreover, it can be obtained from (36) that

$$
\begin{aligned}
\Delta V_{i}(k) & =s_{i}^{2}(k+1)-s_{i}^{2}(k) \leq-\mu^{2} \Longleftrightarrow \\
s_{i}^{2}(k+1) & \leq s_{i}^{2}(k)-\varepsilon^{2} T^{2} \leq s_{i}^{2}(k-1)-2 \mu^{2} \leq \cdots \\
& \leq s_{i}^{2}(0)-(k+1) \mu^{2} .
\end{aligned}
$$


It means that if

$$
s_{i}^{2}(0)-(k+1) \mu^{2}=\eta^{2},
$$

then $\left|s_{i}(k+1)\right| \in \Omega$.

However, the solution of (38) may not be an integer, so we denote $m_{i}=\left(s_{i}^{2}(0)-\eta^{2}\right) / \mu^{2}$ as the real number solution of (38). Then we can say that after at most $m_{i}^{*}=\left[m_{i}\right]+1$ steps, the $i$ th sliding mode state will enter the sliding surface neighborhood region $\boldsymbol{\Omega}$.

Proposition 8. Denote $\varphi=\max \left\{\left(\delta^{*} / \varepsilon T\right)^{1 / \alpha},(\varepsilon T /(1-\right.$ $\left.q T))^{1 /(1-\alpha)}\right\}$, and then there is $\delta^{*} \leq \varepsilon T \varphi^{\alpha} \leq(1-q T) \varphi$.

Lemma 9. For functions in the form:

$$
\eta(\sigma)=1+\sigma^{\sigma /(1-\sigma)}-\sigma^{1 /(1-\sigma)},
$$

where $0<\sigma<1$, there exists $1<\eta(\sigma)<2$, and for any parameter $x \in[0,1]$, the following relation holds [33]:

$$
z \eta(\sigma)-z^{\sigma} \eta^{\sigma}(\sigma)+\eta(\sigma)-1 \geq 0 .
$$

Proof of Problem (ii). When $s_{i}(k) \in \boldsymbol{\Omega}$, it means $-\eta \leq s_{i}(k) \leq$ $\eta$. We can define a new parameter $-1 \leq \theta \leq 1$ such that $s_{i}(k)=$ $\theta \cdot \eta=\theta \cdot \xi(\alpha) \varphi$. The following two cases are discussed.

(1) When $0 \leq \theta \leq 1$, according to (26), we have

$$
\begin{aligned}
s_{i}(k+1)= & (1-q T) \theta \cdot \xi(\alpha) \varphi-\varepsilon T \theta^{\alpha} \xi^{\alpha}(\alpha) \varphi^{\alpha} \\
& +w_{i} \delta_{i} .
\end{aligned}
$$

First, we will prove that $s_{i}(k+1) \leq \eta$.

According to Proposition 8 and Assumption 6, there is

$$
s_{i}(k+1) \leq(1-q T) \theta \cdot \xi(\alpha) \varphi+\delta^{*}\left(1-\theta^{\alpha} \xi^{\alpha}(\alpha)\right) .
$$

If $\theta \xi(\alpha) \geq 1$, we have

$$
s_{i}(k+1) \leq(1-q T) \theta \cdot \xi(\alpha) \varphi \leq \xi(\alpha) \varphi=\eta .
$$

If $0 \leq \theta \xi(\alpha)<1$, we have

$$
\begin{aligned}
s_{i}(k+1) & \leq(1-q T) \varphi\left(1+\theta \cdot \xi(\alpha)-\theta^{\alpha} \xi^{\alpha}(\alpha)\right) \\
& \leq(1-q T) \varphi \leq \xi(\alpha) \varphi=\eta .
\end{aligned}
$$

Second, we will prove that $s_{i}(k+1) \geq-\eta$.

According to Proposition 8, we have

$$
\begin{aligned}
s_{i}(k+1) & \geq(1-q T) \theta \cdot \xi(\alpha) \varphi-\varepsilon T \theta^{\alpha} \xi^{\alpha}(\alpha) \varphi^{\alpha}-\delta^{*} \\
& \geq(1-q T) \varphi\left[\theta \xi(\alpha)-\theta^{\alpha} \xi^{\alpha}(\alpha)-1\right] .
\end{aligned}
$$

If $\theta \xi(\alpha) \geq 1$, since $0<\alpha<1$, we have

$$
s_{i}(k+1) \geq-(1-q T) \varphi \geq-\xi(\alpha) \varphi=-\eta .
$$

If $0 \leq \theta \xi(\alpha)<1$, we have

$$
\begin{aligned}
s_{i}(k+1) & \geq(1-q T) \varphi\left[\theta \xi(\alpha)-\theta^{\alpha} \xi^{\alpha}(\alpha)-1\right] \\
& \geq-\xi(\alpha)(1-q T) \varphi \geq-\xi(\alpha) \varphi=-\eta
\end{aligned}
$$

(2) When $-1 \leq \theta \leq 0$, according to (26), we have

$$
\begin{aligned}
s_{i}(k+1)= & -(1-q T)|\theta \cdot \xi(\alpha)| \varphi+\varepsilon T|\theta \xi(\alpha)|^{\alpha} \varphi^{\alpha} \\
& +w_{i} \delta_{i} .
\end{aligned}
$$

Firstly, we will prove that $s_{i}(k+1) \leq \eta$.

According to Proposition 8, we have

$$
\begin{aligned}
s_{i}(k+1)= & -(1-q T)|\theta \cdot \xi(\alpha)| \varphi+\varepsilon T|\theta \xi(\alpha)|^{\alpha} \varphi^{\alpha} \\
& +w_{i} \delta_{i} \\
\leq & -(1-q T)|\theta \cdot \xi(\alpha)| \varphi \\
& +(1-q T) \varphi|\theta \xi(\alpha)|^{\alpha}+\delta^{*} \\
\leq & (1-q T) \varphi\left[-|\theta \cdot \xi(\alpha)|+|\theta \xi(\alpha)|^{\alpha}+1\right] .
\end{aligned}
$$

If $|\theta \xi(\alpha)| \geq 1$, since $0<\alpha<1$, we have

$$
s_{i}(k+1) \leq(1-q T) \varphi \leq \xi(\alpha) \varphi=\eta .
$$

If $0 \leq|\theta \xi(\alpha)|<1$, according to Lemma 9, we have

$$
s_{i}(k+1) \leq(1-q T) \varphi \xi(\alpha) \leq \varphi \xi(\alpha)=\eta .
$$

Second, we will prove that $s_{i}(k+1) \geq-\eta$.

According to Proposition 8, we have

$$
\begin{aligned}
s_{i}(k+1)= & -(1-q T)|\theta \cdot \xi(\alpha)| \varphi+\varepsilon T|\theta \xi(\alpha)|^{\alpha} \varphi^{\alpha} \\
& +w_{i} \delta_{i} \\
\geq & -(1-q T)|\theta \cdot \xi(\alpha)| \varphi+|\theta \xi(\alpha)|^{\alpha} \delta^{*} \\
& -\left|w_{i} \delta_{i}\right| \\
= & (1-q T)|\theta \cdot \xi(\alpha)| \varphi \\
& +\delta^{*}\left(|\theta \xi(\alpha)|^{\alpha}-1\right) .
\end{aligned}
$$

If $|\theta \xi(\alpha)| \geq 1$, we have

$$
s_{i}(k+1) \geq-(1-q T)|\theta \cdot \xi(\alpha)| \varphi \geq-\xi(\alpha) \varphi=-\eta .
$$

If $0 \leq|\theta \xi(\alpha)|<1$, we have

$$
\begin{aligned}
s_{i}(k+1) & \geq-(1-q T) \varphi\left[|\theta \cdot \xi(\alpha)|-|\theta \xi(\alpha)|^{\alpha}+1\right] \\
& \geq-(1-q T) \varphi \geq \xi(\alpha) \varphi=-\eta .
\end{aligned}
$$

To this end, we can conclude that when $-\eta \leq s_{i}(k) \leq \eta$, we have $-\eta \leq s_{i}(k+1) \leq \eta$, which means $s_{i}(k+1) \in \mathbf{\Omega}$.

Remark 10. For discrete-time systems, the accurate arrival of sliding mode state on the sliding surface is hard to realize, so to totally eliminate chattering is actually not possible in the discrete-time systems. This paper uses $\operatorname{sig}^{\alpha}(\boldsymbol{s}(k))$ to replace the sign function to avoid the sharp switching of control input to suppress chattering. The closer the sliding mode state to the sliding surface, the lower the chattering that will be obtained. It needs to be pointed out that the saying "chattering-free" here is just a conventional way to describe such chattering suppression methods [32, 34, 35]. 
3.3. Design of Multiple-Model Based Compensator. Traditionally, if time delay information cannot be foreseen by the controller, there can only be two control input compensation strategies, i.e., zero-input or hold-input. For zero-input strategy, it means if the controller output $\overline{\boldsymbol{u}}(k)$ does not arrive on time, then we let control input $\boldsymbol{u}(k)=0$. However, when time delay happens frequently, this compensation strategy will result in very poor dynamic performance. And for holdinput strategy, it means control signal will remain unchanged unless new control signal arrives, but this may cause serious overshoot when long time delay exists. With a focus on the above problems, a new compensation strategy is proposed, which also comes from the idea of hold-input strategy but, compared with traditional hold-input strategy, it uses different system models to calculate control input signal for different equivalent time delay conditions. And to overcome the problem that controller cannot foresee the controlleractuator equivalent time delay, all possible models relating to different equivalent time delay $\tau(k)$ are established in the controller. Then, at each controlling period, instead of outputting one control signal, the controller will calculate and output a sequence of different control signals $\bar{U}(k)=$ $\left[\overline{\boldsymbol{u}}(k)_{\tau=0}, \overline{\boldsymbol{u}}(k)_{\tau=1}, \ldots, \overline{\boldsymbol{u}}(k)_{\tau=\bar{\tau}+\bar{\rho}}\right]$ based on different models and the controller output $\bar{U}(k)$ will be put in one data packet with a time stamp. Also at each controlling period, the $\mathrm{ZOH}$ will output a signal sequence packet $\boldsymbol{U}(k)$ based on the time stamp information, which means $\boldsymbol{U}(k)=\overline{\boldsymbol{U}}(k)$ for $\tau(k)=0$, or $\boldsymbol{U}(k)=\overline{\boldsymbol{U}}(k-1)$ for $\tau(k)=1$, etc. Finally, the compensator will decide which control signal of $\boldsymbol{U}(k)$ to use according to the time stamp information; i.e., if $\boldsymbol{U}(k)=\overline{\boldsymbol{U}}(k)$, it will use the first signal of $\boldsymbol{U}(k)$, and if $\boldsymbol{U}(k)=\overline{\boldsymbol{U}}(k-1)$, it will use the second signal of $\boldsymbol{U}(k)$, etc.

In this way, even though there is no newly received control signal by the $\mathrm{ZOH}$, the actuator can still get an updated control signal from the compensator and, unlike the traditional hold-input strategy, the updated control input signal has already taken into account the state changes of the former sampling period.

Based on the above discussion, the algorithm of the compensator can be described as

$$
\boldsymbol{u}(k)=\boldsymbol{U}(k) \boldsymbol{g}(\tau(k))=\overline{\boldsymbol{U}}(k-\tau(k)) \boldsymbol{g}(\tau(k)),
$$

where the compensation signal choosing function $\boldsymbol{g}(x)$ is defined as

$$
\begin{aligned}
& \boldsymbol{g}(x)=\left[g_{0}, g_{1}, \ldots, g_{i}, \ldots, g_{\bar{\tau}+\bar{\rho}}\right]^{\mathrm{T}}, \\
& g_{i}= \begin{cases}1, & i=x \\
0, & i \neq x .\end{cases}
\end{aligned}
$$

To show the relationship between actuator input and controller output more clearly, the following illustrations are given:

$$
\boldsymbol{u}(k)= \begin{cases}\overline{\boldsymbol{U}}(k) \cdot\left[\begin{array}{lll}
1 & 0 & \ldots
\end{array}\right]^{\mathrm{T}}=\overline{\boldsymbol{u}}(k)_{\tau=0}, & \tau=0 \\
\overline{\boldsymbol{U}}(k-1) \cdot\left[\begin{array}{lll}
0 & 1 & 0 \ldots 0
\end{array}\right]^{\mathrm{T}}=\overline{\boldsymbol{u}}(k-1)_{\tau=1}, & \tau=1 \\
\vdots & \vdots \\
\overline{\boldsymbol{U}}(k-\tau(k)) \cdot\left[\begin{array}{lll}
\underbrace{0 \ldots 0}_{\tau(k)} & 1 & 0 \ldots 0
\end{array}\right]^{\mathrm{T}}=\overline{\boldsymbol{u}}(k-\tau(k))_{\tau=\tau(k)}, & \tau=\tau(k) \\
\vdots & \vdots \\
\overline{\boldsymbol{U}}(k-\bar{\tau}-\bar{\rho}) \cdot\left[\begin{array}{lll}
0 \ldots 0 & 1
\end{array}\right]^{\mathrm{T}}=\overline{\boldsymbol{u}}(k-\bar{\tau}-\bar{\rho})_{\tau=\bar{\tau}+\bar{\rho}}, & \tau=\bar{\tau}+\bar{\rho} .\end{cases}
$$

In conclusion, the steps of designing this chattering-fee sliding mode controller are given below.

Step 1. Obtain the system's time delay and packet dropout characteristics through experiments and then get the transition probability matrix (6) based on the proposed modeling method.

Step 2. Obtain the system sliding mode regular form (16a) and (16b) through linear transformation $\boldsymbol{z}=\boldsymbol{T} \overline{\boldsymbol{x}}$, where the definition of nonsingular matrix $\boldsymbol{T} \in \mathrm{R}^{n \times n}$ is given by (14) and (15).

Step 3. Design the classic linear sliding surface (17), whose parameter $\boldsymbol{C}=\left[\begin{array}{ll}\boldsymbol{C}_{1} & \boldsymbol{I}\end{array}\right]$ can be determined by pole placement method.
Step 4. Design the chattering-free reaching law (20) and choose appropriate reaching law parameters $q, \varepsilon$, and $\alpha$ that satisfy $0<q T<1,0<\varepsilon T<1,0<\alpha<1$.

Step 5. Design the sliding mode controller (22) according to reaching law (20).

Step 6. Design the multiple-model based compensator (57) based on the upper bound of long time delay and consecutive packet dropout.

\section{Simulation Example}

In this section, a simulation example is given to illustrate the effectiveness of the proposed method. Consider the 
following NCS in the form of (1), which comes from a type of aeroengine control system [36]. System parameters are initialed as follows:

$$
\begin{aligned}
\boldsymbol{A} & =\left[\begin{array}{ccc}
-0.9 & 0.1 & -0.2 \\
-0.7 & 0.9 & -0.5 \\
0.4 & -0.8 & 0.6
\end{array}\right], \\
\boldsymbol{B} & =\left[\begin{array}{cc}
0.2 & 0.1 \\
0.1 & 0.4 \\
0.3 & -0.1
\end{array}\right], \\
\boldsymbol{x} & =\left[\begin{array}{lll}
n_{L} & n_{H} & p_{3}
\end{array}\right]^{\mathrm{T}}, \\
\boldsymbol{u} & =\left[\begin{array}{ll}
m_{\mathrm{f}} & A_{8}
\end{array}\right]^{\mathrm{T}},
\end{aligned}
$$

where $n_{\mathrm{L}}$ is low pressure rotor speed; $n_{\mathrm{H}}$ is high pressure rotor speed; $p_{3}$ is compressor exit total pressure; $m_{\mathrm{f}}$ is fuel flow; and $A_{8}$ is critical section area of nozzle.

System sampling period is set as $T=20 \mathrm{~ms}$. The initial system state is $\boldsymbol{x}(0)=\left[\begin{array}{lll}-0.9 & 0.5 & -0.7\end{array}\right]^{T}$. The disturbance is chosen as

$$
\boldsymbol{d}(k)=\boldsymbol{B} \boldsymbol{\omega}(k)=\boldsymbol{B} \boldsymbol{N} \zeta(k),
$$

where $\zeta(k)=1.2 \sin (0.5 \pi \cdot k)$ and $\boldsymbol{N}=\left[\begin{array}{ll}0.5 & 0.3\end{array}\right]^{\mathrm{T}}$.

The sliding function parameter $\boldsymbol{C}=\left[\boldsymbol{C}_{1} \boldsymbol{I}\right]$ can be designed based on pole placement method, and by choosing the pole location of closed-loop system (16a) as [-0.5], it can be obtained that $C_{1}=\left[\begin{array}{lll}2.8539 & -0.0238\end{array}\right]^{\mathrm{T}}$, and thus $\boldsymbol{C}=$ $\left[\begin{array}{ccc}2.8539 & 1 & 0 \\ -0.0238 & 0 & 1\end{array}\right]$.

Then choose the reaching law parameters as $q=10, \varepsilon=$ 0.5 , and $\alpha=0.5$.

For long time delay and packet dropout, it is defined that $\bar{\tau}=2$ and $\bar{\rho}=2$, and the probability transition matrix is given below:

$$
\Pi_{\bar{\tau}, \bar{\rho}}=\left[\begin{array}{ccccc}
0.7 & 0.3 & 0 & 0 & 0 \\
0.6 & 0.2 & 0.2 & 0 & 0 \\
0.4 & 0.2 & 0.2 & 0.2 & 0 \\
0.3 & 0.2 & 0.2 & 0 & 0.3 \\
0.4 & 0.4 & 0.2 & 0 & 0
\end{array}\right] .
$$

Based on matrix $\Pi_{\bar{\tau}, \bar{\rho}}$, a Markov chain typed equivalent time delay distribution is shown in Figure 2, which simulates the actual time delay and packet dropout condition of a networked control system during 100 sampling periods.

It can be seen from Figure 2 that $\tau(k)=3$ occurs four times, and $\tau(k)=4$ does not occur. Accordingly, we can see that, due to the use of $\mathrm{ZOH}$ and the application of the proposed new modeling method, consecutive packet dropout does not really affect the system in this case even though it does exist. However, the effect of consecutive packet dropout may become noticeable if the upper bound of consecutive packet dropout is larger than two, but there is still no doubt that the proposed method greatly suppresses the effect of it.

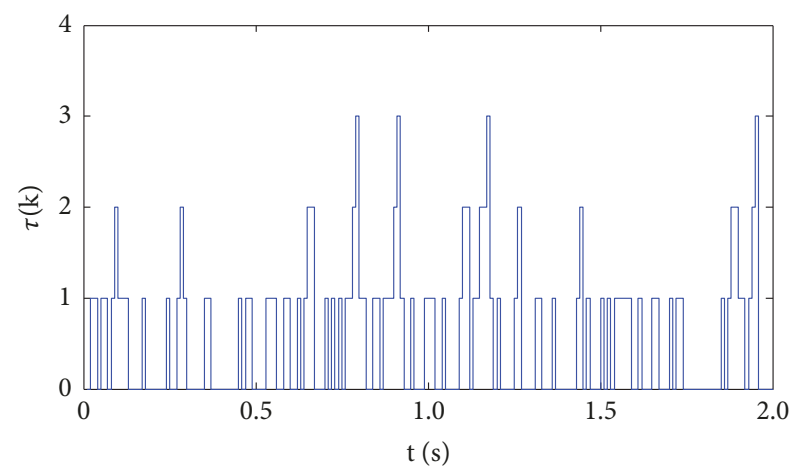

FIGURE 2: Distribution of equivalent time delay.

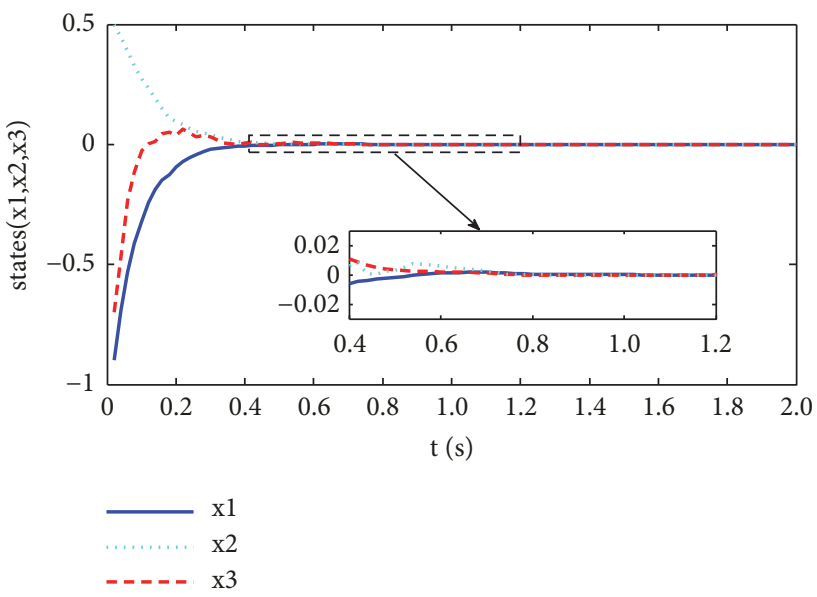

FIGURE 3: System state trajectory with the proposed controller.

Simulation results obtained by using the proposed chattering-free SMC are shown in Figures 3-5. To show the superiority of the proposed controller in chattering suppression, another controller is applied which is the classic reaching law based SMC proposed by Gao [37]. The same compensation strategy is also used in this controller. The results obtained are shown in Figures 6-8. The comparison of Figure 3 with Figure 6 reveals that the system states converge in less time with lower chattering in the case of the proposed controller as compared to the classic reaching law based SMC. The comparison of Figure 4 with Figure 7, and Figure 5 with Figure 8 gives the same conclusion in control input and sliding function.

Moreover, to testify the effectiveness of the proposed multiple-model based compensator, another controller has been constructed which has the same chattering-free reaching law with the proposed controller but without compensator. Instead, the traditional hold-input compensation strategy is used. First, the condition of $\bar{\tau}=2$ and $\bar{\rho}=$ 2 is considered and the response of system state $x_{1}$ with two controllers is shown in Figure 9, where controller 1 is the proposed controller and controller 2 is the proposed controller without compensator. It can be seen that there is no essential difference between the response curves of $x_{1}$ with two controllers because the upper bound of time delay 

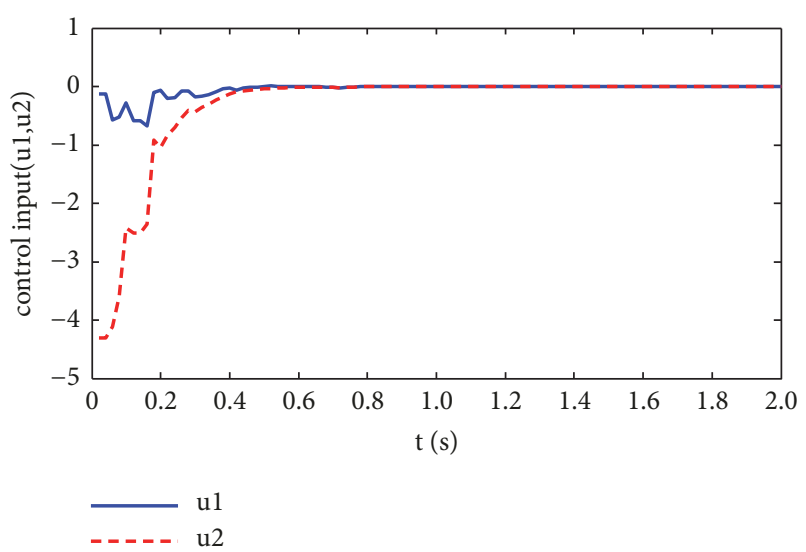

FIGURE 4: Control input trajectory with the proposed controller.

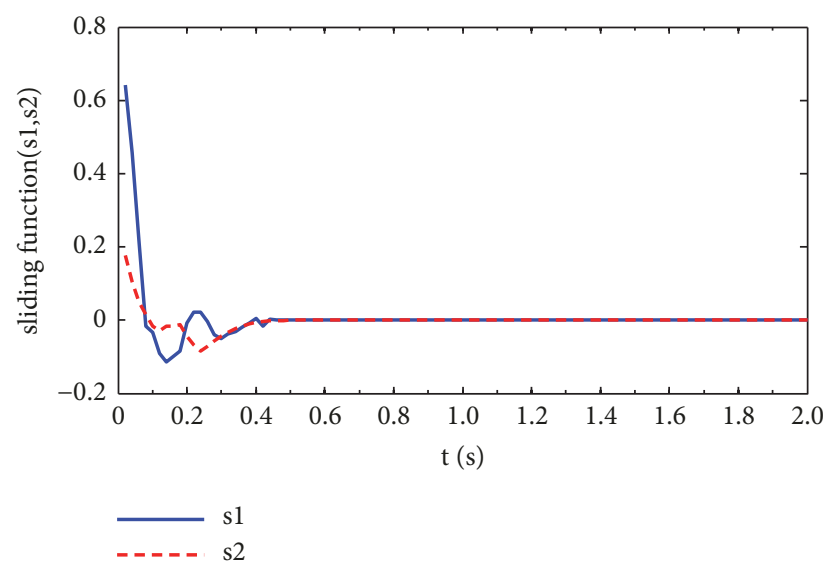

FIgURE 5: Sliding function trajectory with the proposed controller.

and packet dropout is small and the hold-input strategy is sufficient for compensation. Then Figures 10 and 11 depict the response curves of $x_{1}$ on conditions $\bar{\tau}=3, \bar{\rho}=3$ and $\bar{\tau}=4, \bar{\rho}=4$, respectively. It is obvious that the performance of controller 2 (proposed controller without compensator) deteriorates sharply when the upper bound of time delay and packet dropout grows, while the proposed controller suffers little from the changes.

\section{Conclusion}

This paper proposes a chattering-free sliding mode controller with multiple-model based delay compensator for NCSs with long time delay and consecutive packet dropout. By proposing a new modeling method, long time delay and consecutive packet dropout can be modeled in a unified model described by one Markov chain, which not only simplifies the system model but also makes the controller designed more suitable for practical use. To the best of the authors' knowledge, this modeling method for time delay and packet dropout has not been reported by any existing literature and it is the use of this model that makes the proposed compensator function well. A chattering-free sliding mode reaching law is then

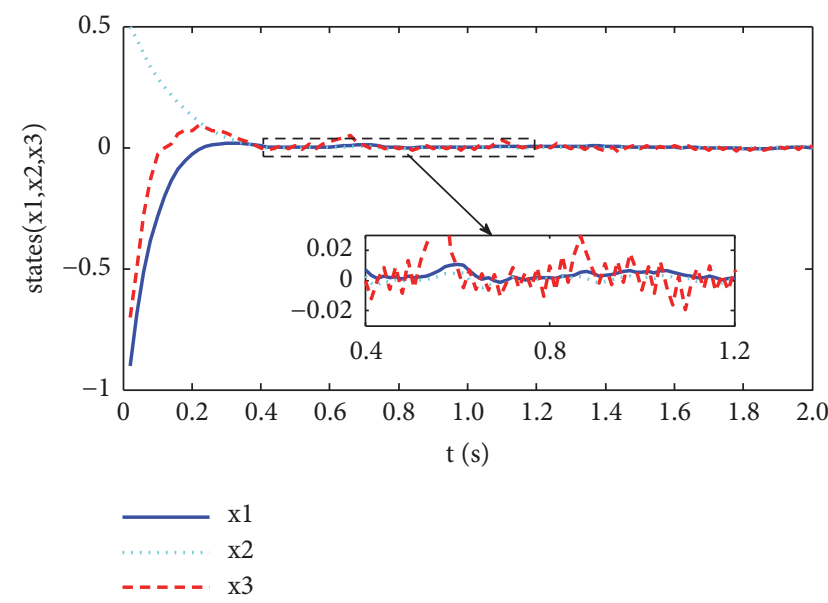

FIGURE 6: System state trajectory with classic reaching law based SMC.

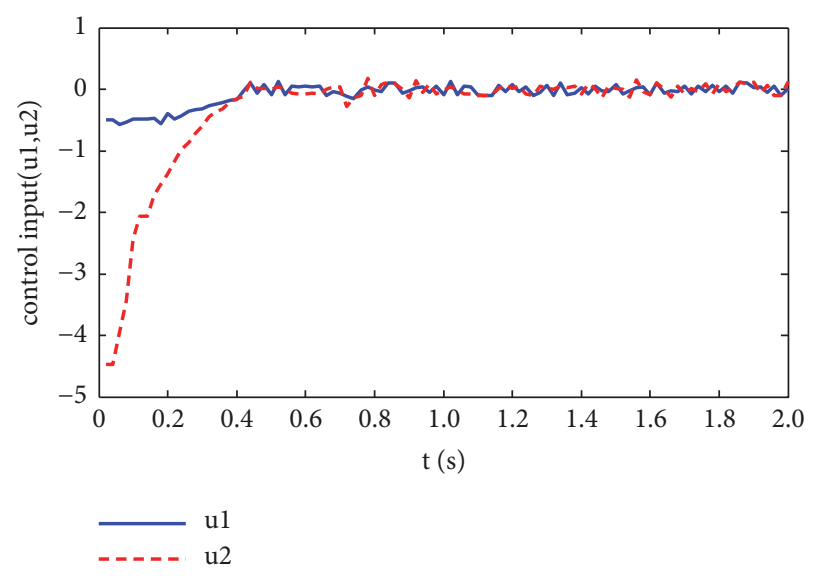

FIgURE 7: Control input trajectory with the classic reaching law based SMC

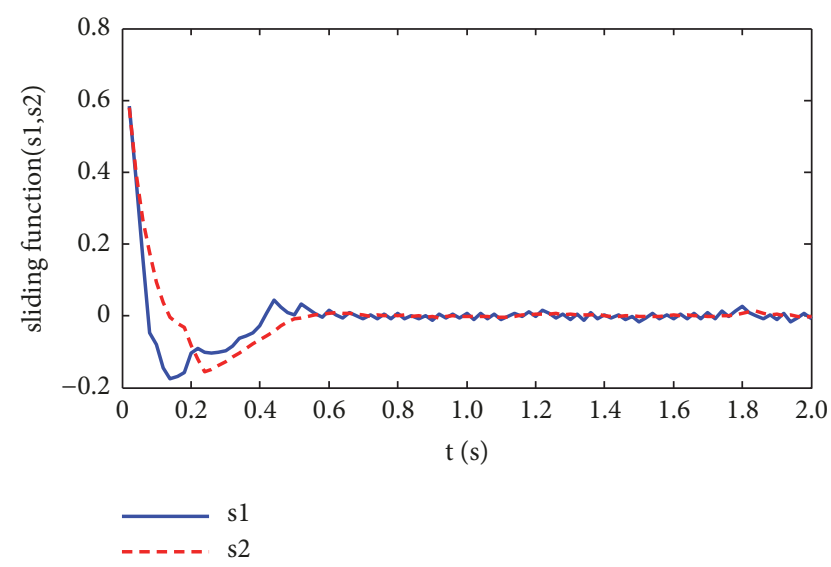

FIGURE 8: Sliding function trajectory with the classic reaching law based SMC. 


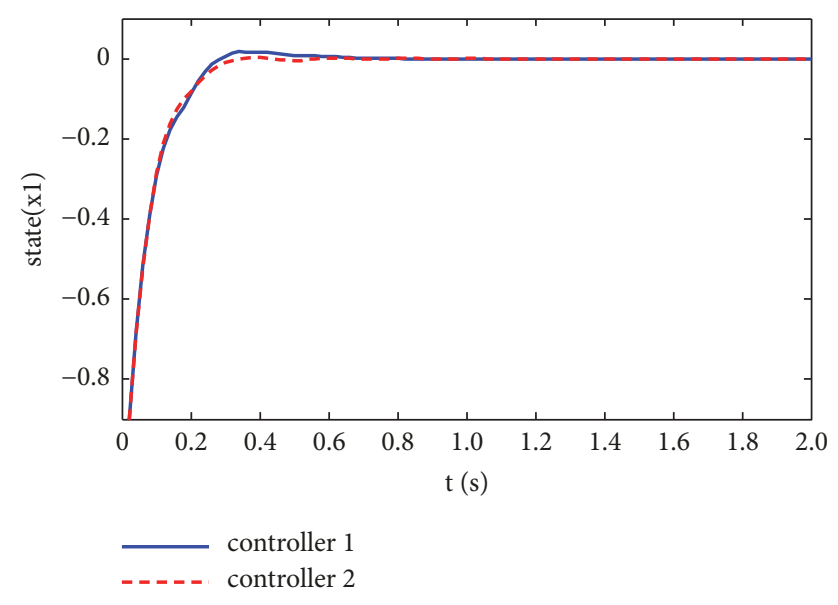

FIgURE 9: Trajectory of state $x_{1}$ with two controllers on condition $\bar{\tau}=2, \bar{\rho}=2$.

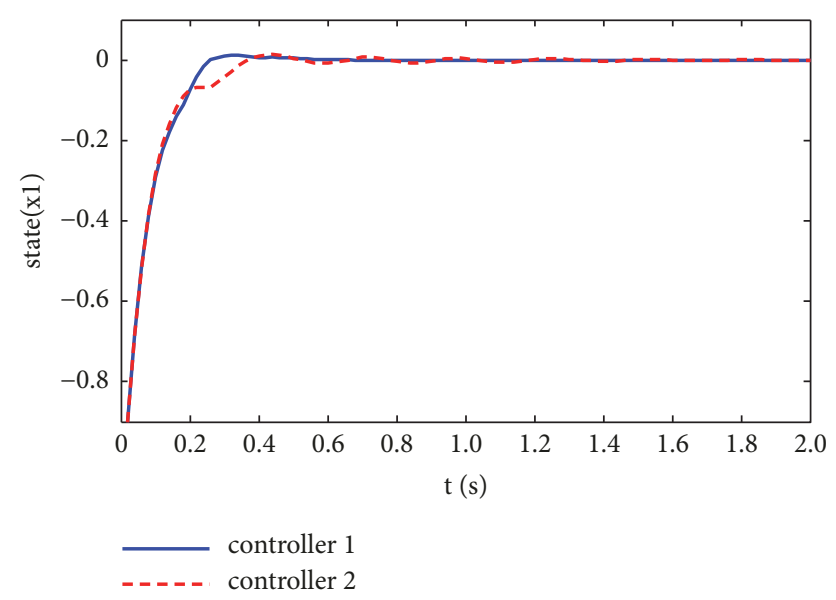

FIgURE 10: Trajectory of state $x_{1}$ with two controllers on condition $\bar{\tau}=3, \bar{\rho}=3$.

proposed and it is also the first time that such chatteringfree reaching law is used for multiple-input discrete-time systems. To overcome the problem that controller-actuator channel network condition cannot be foreseen by controller, a new compensation strategy is proposed and a multiple-model based compensator is constructed. Finally, a simulation example is given and it shows that the proposed method can effectively overcome the effect of time delay and packet dropout as well as disturbance and can make the system states converge to the origin quickly without noticeable chattering. Therefore, the proposed method serves as a suitable choice for uncertain NCSs with long time delay and consecutive packet dropout. In our future research, we will work on testing the proposed method in a practical system so that the theory proposed can be more completed.

\section{Data Availability}

The data used to support the findings of this study are already included within the article, which have been given clearly at the beginning of the section "Simulation Example".

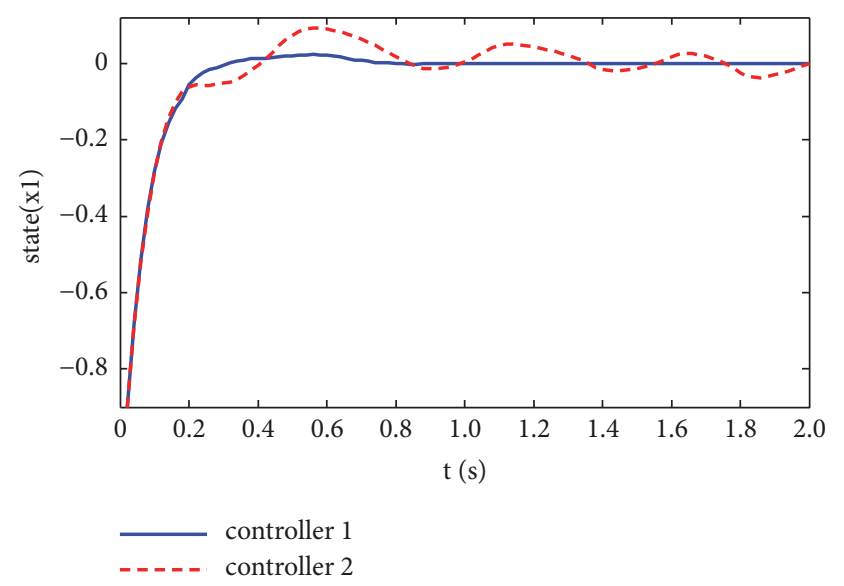

FIGURE 11: Trajectory of state $x_{1}$ with two controllers on condition $\bar{\tau}=4, \bar{\rho}=4$.

\section{Conflicts of Interest}

The authors declare that they have no conflicts of interest.

\section{Acknowledgments}

This work was supported by the National Natural Science Foundation of China (51506221, 51476187, and 51606219).

\section{References}

[1] S. Yan, G. Zhang, T. Li, M. Shen, and L. Li, " $\mathrm{H}_{\infty}$ static output control of discrete-time networked control systems with an event-triggered scheme," Circuits, Systems and Signal Processing, vol. 37, no. 2, pp. 553-568, 2018.

[2] D. Almakhles, A. K. Swain, A. Nasiri, and N. Patel, "An adaptive two-level quantizer for networked control systems," IEEE Transactions on Control Systems Technology, vol. 25, no. 3, pp. 1084-1091, 2017.

[3] A. Argha, L. Li, and S. W. Su, " $\mathrm{H}_{2}$ based optimal sparse sliding mode control for networked control systems," International Journal of Robust and Nonlinear Control, vol. 28, no. 1, pp. 16-30, 2018.

[4] C. Yuan and F. Wu, "Delay scheduled impulsive control for networked control systems," IEEE Transactions on Control of Network Systems, vol. 4, no. 3, pp. 587-597, 2017.

[5] D. Shah and A. Mehta, "Fractional delay compensated discretetime SMC for networked control system," Digital Communications and Networks, vol. 3, no. 2, pp. 112-117, 2017.

[6] X. Li and S. Sun, " $H_{\infty}$ control for networked stochastic nonlinear systems with randomly occurring sensor saturations, multiple delays and packet dropouts," IET Control Theory \& Applications, vol. 11, no. 17, pp. 2954-2963, 2017.

[7] D. H. Shah and A. Mehta, "Discrete-time sliding mode controller subject to real-time fractional delays and packet losses for networked control system," International Journal of Control, Automation and Systems, vol. 15, no. 6, pp. 2690-2703, 2017.

[8] W. Wu, "Fault-tolerant control of uncertain non-linear networked control systems with time-varying delay, packet dropout and packet disordering," IET Control Theory \& Applications, vol. 11, no. 7, pp. 973-984, 2017. 
[9] C. Tan and H. Zhang, "Necessary and sufficient stabilizing conditions for networked control systems with simultaneous transmission delay and packet dropout," IEEE Transactions on Automatic Control, vol. 62, no. 8, pp. 4011-4016, 2017.

[10] J. Chen, "Random fuzzy slow state feedback control for networked two time-scale nonlinear systems with time-delay and data packets dropout," International Journal of Modelling, Identification and Control, vol. 28, no. 1, pp. 1-13, 2017.

[11] J. Gao, H. Su, and J. Chu, "Robust stability analysis of networked control systems," Control Theory and Technology, vol. 7, no. 3, pp. 301-306, 2009.

[12] M. Yu, X. Yuan, and W. Xiao, "A switched system approach to robust stabilization of networked control systems with multiple packet transmission," Asian Journal of Control, vol. 17, no. 4, pp. 1415-1423, 2015.

[13] K. Halder, S. Das, S. Dasgupta, S. Banerjee, and A. Gupta, "Controller design for networked control systems-an approach based on $L_{2}$ induced norm," Nonlinear Analysis: Hybrid Systems, vol. 19, pp. 134-145, 2016.

[14] Y. Wu, T. Liu, Y. Wu, and Y. Zhang, " $\mathrm{H}_{\infty}$ output tracking control for uncertain networked control systems via a switched system approach," International Journal of Robust and Nonlinear Control, vol. 26, no. 5, pp. 995-1009, 2016.

[15] D. Zhang, Q.-G. Wang, L. Yu, and Q.-K. Shao, " $H_{\infty}$ filtering for networked systems with multiple time-varying transmissions and random packet dropouts," IEEE Transactions on Industrial Informatics, vol. 9, no. 3, pp. 1705-1716, 2013.

[16] Y. Zhang and H. Fang, "Stabilization of nonlinear networked systems with sensor random packet dropout and time-varying delay," Applied Mathematical Modelling, vol. 35, no. 5, pp. 22532264, 2011.

[17] Z. Hu, F. Deng, M. Xing, and J. Li, "Modeling and control of Itô stochastic networked control systems with random packet dropouts subject to time-varying sampling," Institute of Electrical and Electronics Engineers Transactions on Automatic Control, vol. 62, no. 8, pp. 4194-4201, 2017.

[18] M. Azadegan and M. T. Beheshti, "Robust stability and stabilization of TCP-networked control systems with multiple delay system modeling," Asian Journal of Control, vol. 19, no. 3, pp. 1034-1045, 2017.

[19] D. Wu, J. Wu, and S. Chen, "Separation principle for networked control systems with multiple-packet transmission," IET Control Theory \& Applications, vol. 5, no. 3, pp. 507-513, 2011.

[20] J. Liu and X. Ruan, "Networked iterative learning control for discrete-time systems with stochastic packet dropouts in input and output channels," Advances in Difference Equations, vol. 53, pp. 1-21, 2017.

[21] Y. Shi and B. Yu, "Output feedback stabilization of networked control systems with random delays modeled by Markov chains," IEEE Transactions on Automatic Control, vol. 54, no. 7, pp. 1668-1674, 2009.

[22] J. Dong and W. Kim, "Markov-chain-based output feedback control for stabilization of networked control systems with random time delays and packet losses," International Journal of Control, Automation, and Systems, vol. 10, no. 5, pp. 1013-1022, 2012.

[23] H. Li, H. Yang, F. Sun, and Y. Xia, "Sliding-mode predictive control of networked control systems under a multiple-packet transmission policy," IEEE Transactions on Industrial Electronics, vol. 61, no. 11, pp. 6234-6243, 2014.
[24] J. Wu, L. Zhang, and T. Chen, "Model predictive control for networked control systems," International Journal of Robust and Nonlinear Control, vol. 19, no. 9, pp. 1016-1035, 2009.

[25] L. Qiu, Q. Luo, F. Gong, S. Li, and B. Xu, "Stability and stabilization of networked control systems with random time delays and packet dropouts," Journal of The Franklin Institute, vol. 350, no. 7, pp. 1886-1907, 2013.

[26] B. Lian, Q. Zhang, and J. Li, "Sliding mode control for nonlinear networked control systems subject to packet disordering via prediction method," IET Control Theory \& Applications, vol. 11, no. 17, pp. 3079-3088, 2017.

[27] D. Wang, J. Wang, and W. Wang, "Output feedback control of networked control systems with packet dropouts in both channels," Information Sciences, vol. 221, pp. 544-554, 2013.

[28] Y. Xia, Z. Zhu, C. Li, H. Yang, and Q. Zhu, "Robust adaptive sliding mode control for uncertain discrete-time systems with time delay," Journal of The Franklin Institute, vol. 347, no. 1, pp. 339-357, 2010.

[29] M. Kchaou and S. A. Ahmadi, "Robust $\mathrm{H}_{\infty}$ control for nonlinear uncertain switched descriptor systems with time delay and nonlinear input: a sliding mode approach," Complexity, vol. 2017, Article ID 1027909, 14 pages, 2017.

[30] H. B. Du, X. P. Chen, G. H. Wen, X. H. Yu, and J. H. Lv, "Discretetime fast terminal sliding mode control for permanent magnet linear motor," IEEE Transactions on Industrial Electronics, 2018.

[31] F. Yang, W. Wang, Y. Niu, and Y. Li, “Observer-based H $\infty$ control for networked systems with consecutive packet delays and losses," International Journal of Control, Automation, and Systems, vol. 8, no. 4, pp. 769-775, 2010.

[32] H. Du, X. Yu, M. Z. Chen, and S. Li, "Chattering-free discretetime sliding mode control," Automatica, vol. 68, pp. 87-91, 2016.

[33] S. Li, H. Du, and X. Yu, "Discrete-time terminal sliding mode control systems based on Euler's discretization," Institute of Electrical and Electronics Engineers Transactions on Automatic Control, vol. 5, no. 2, pp. 546-552, 2014.

[34] J.-N. Li, H. Su, Y. Zhang, Z.-G. Wu, and J. Chu, "Chattering free sliding mode control for uncertain discrete time-delay singular systems," Asian Journal of Control, vol. 15, no. 1, pp. 260-269, 2013.

[35] S. Mondal and C. Mahanta, "Chattering free adaptive multivariable sliding mode controller for systems with matched and mismatched uncertainty," ISA Transactions ${ }^{\circledR}$, vol. 52, no. 3, pp. 335-341, 2013.

[36] L. Ren, S. Xie, and J. Peng, "Adaptive robust sliding mode control for aeroengine T-S distributed system," Journal of Propulsion Technology, vol. 37, no. 12, 2016.

[37] W. Gao, Y. Wang, and A. Homaifa, "Discrete-time variable structure control systems," IEEE Transactions on Industrial Electronics, vol. 42, no. 2, pp. 117-122, 1995. 


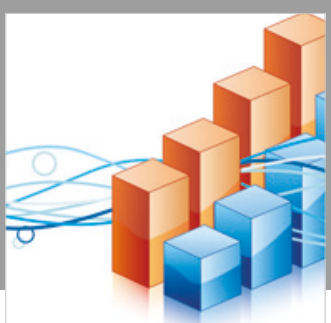

Advances in

Operations Research

\section{-n-m}
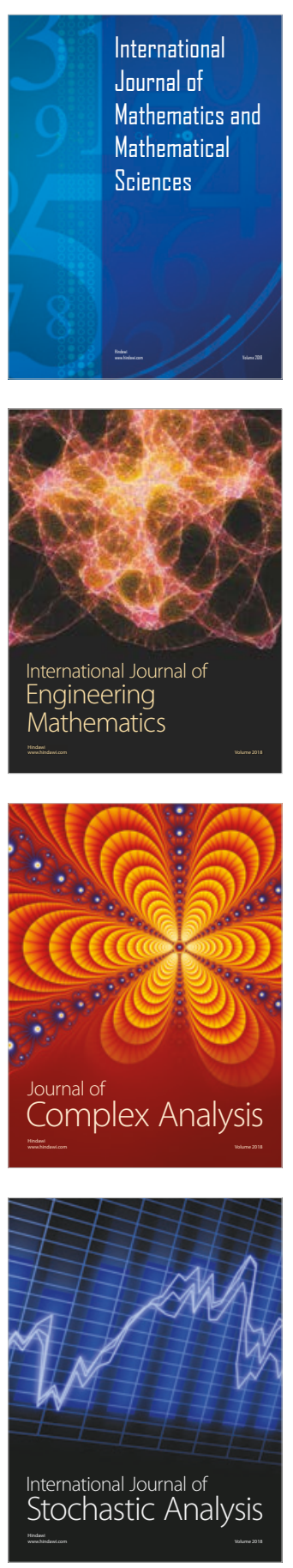
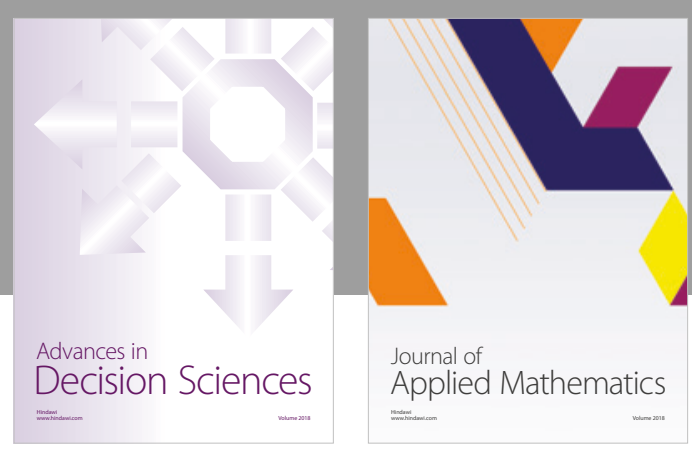

Journal of

Applied Mathematics
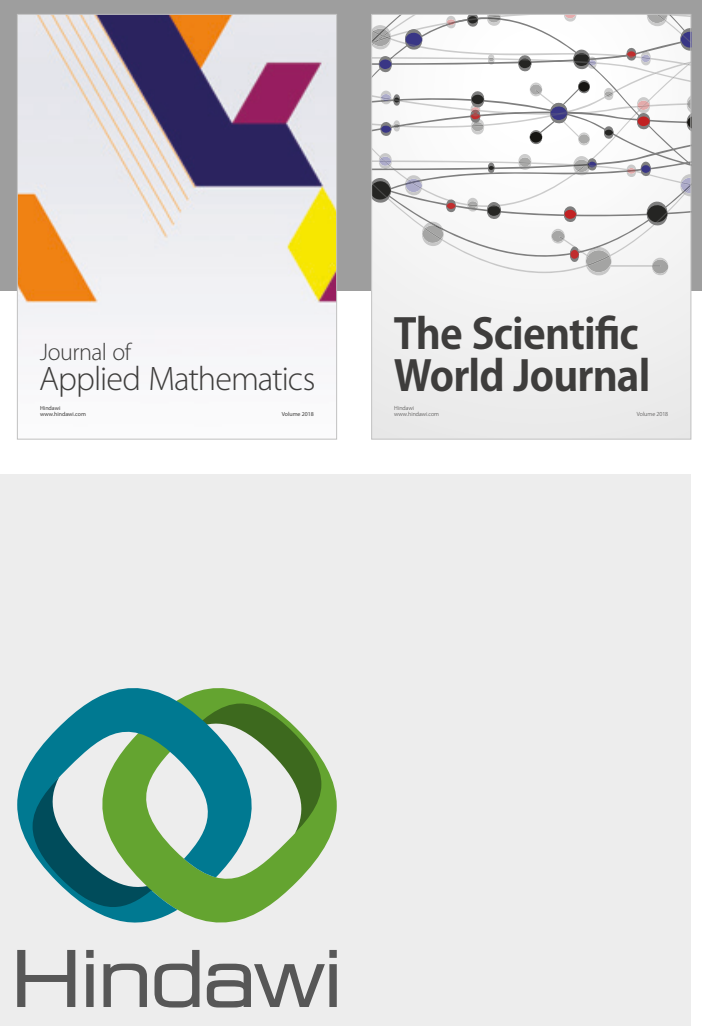

Submit your manuscripts at

www.hindawi.com

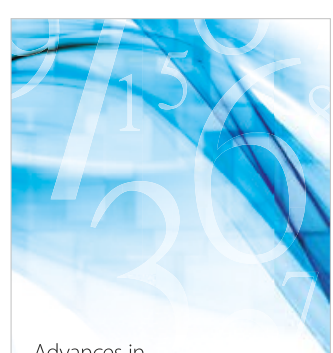

Advances in
Numerical Analysis
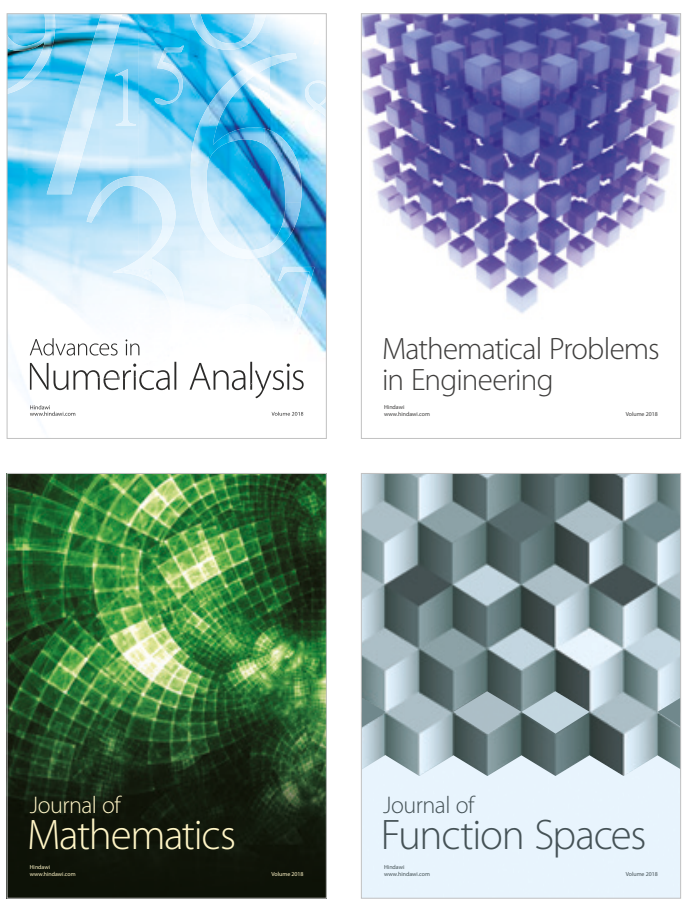

Mathematical Problems in Engineering

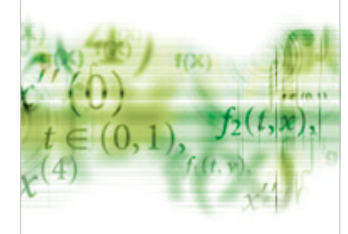

International Journal of

Differential Equations

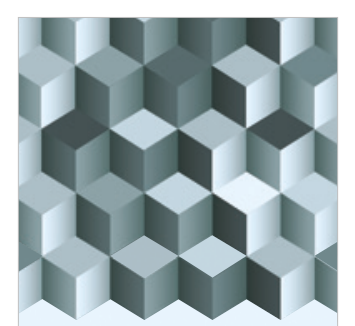

Journal of

Function Spaces
The Scientific

World Journal

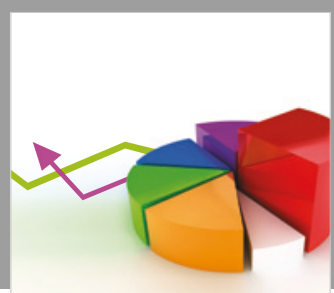

Journal of

Probability and Statistics
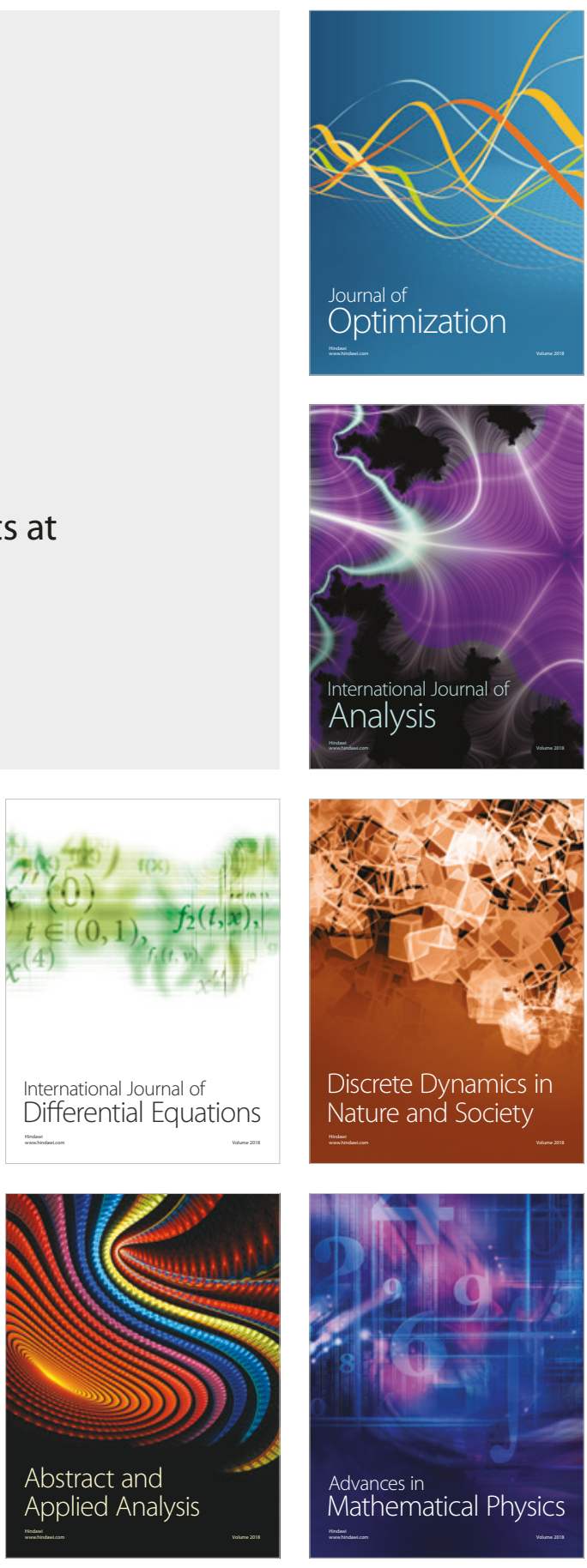